\title{
High-resolution X-ray source with advanced e-beam technology: pushing the resolution limitation for lab-scale NanoCT
}

\author{
Emil Espes and Anasuya Adibhatla
}

Excillum, United States

Since more than ten years, MetalJet sources, based on liquid-metal-jet technology, are successfully operated in many labs over the world. By using a high-speed jet of liquid metal, instead of the traditional solid- or rotating anode, it has been demonstrated that a much higher power can be applied to the anode. Since melting of the anode is thereby no longer a problem as it is already molten, MetalJet has achieved an at least 10x significantly higher brightness than the conventional solid-anode microfocus tube with the X-ray spot size range of 5- $40 \mu \mathrm{m}$. Key applications include X-ray diffraction and scattering, but several publications have also shown very impressive imaging results using liquid-metal-jet technology, especially in phase-contrast imaging and X-ray microscopy.

Driven by needs from scientific research, healthcare and industrial applications, X-ray microscopy has been successfully transferred from synchrotron to laboratory and the spatial resolution has been pushed to sub-micrometer. One way to further improve the resolution is to use the X-ray source with small focal spot. Based on advanced electron beam and target technologies, a state-of-art nanofocus X-ray tube has been developed by Excillum. The NanoTube N2 reaches an isotropic resolution of $150 \mathrm{~nm}$ lines and spaces.

Further unique features include high stability for long-time imaging investigations, continuous switching among different voltage and spot size without change in X-ray spot position, internal electron-beam size measurement and user-defined electron-beam deflection. Furthermore, the front surface of the NanoTube is cone shaped, so that the sample could be mounted as close as possible to the focal spot with standard a CT sample holder.

Typical nanofocus X-ray tubes normally have rather limited flux, which leads to long acquisition times of nanoCT in lab. The NanoTube N2 was launched in 2020 and is currently available at $60 \mathrm{kVp}$, and 110 $\mathrm{kVp}$. The newly released NanoTube N2 $60 \mathrm{kV}$ achieves at least 3x higher flux than its predecessor (NanoTube N1) at $60 \mathrm{kV}$, which means a 3x faster image acquisition. NanoTube N2 $110 \mathrm{kV}$ offers further increase in flux at small spot sizes and a higher emission voltage, enabling larger and/or higher absorbing samples, covering most materials science applications.

Until now the NanoTube has been integrated into different Nano-CT systems for applications of biomedical [1][2][3] and materials science [4], as well as non-destructive testing and industrial inspection. Particularly, the Nano-CT system developed at Fraunhofer IIS, has turned into a commercialized system through ProCon X-ray GmbH.

\section{References}

[1] M. Müller et al., (2017). PNAS 114 (47), 12378-12383 (2017)

[2] S. Ferstl et al., IEEE Transactions on Medical Imaging, 1-1 (2019)

[43] J. Romell et al., Proc. of SPIE 11112, X-ray Nanoimaging: Instruments and Methods IV (2019)

[4] C. Fella et al., Microscopy and Microanalysis, 24(S2), 234-235 (2018) 